PROCEEDINGS OF THE

AMERICAN MATHEMATICAL SOCIETY

Volume 128, Number 9 , Pages $2569-2575$

S 0002-9939(00)05313-2

Article electronically published on February 29, 2000

\title{
UNIFORMLY PERFECT SETS, RATIONAL SEMIGROUPS, KLEINIAN GROUPS AND IFS'S
}

\author{
RICH STANKEWITZ
}

(Communicated by Albert Baernstein II)

\begin{abstract}
We show that the Julia set of a non-elementary rational semigroup $G$ is uniformly perfect when there is a uniform bound on the Lipschitz constants of the generators of $G$. This also proves that the limit set of a non-elementary Möbius group is uniformly perfect when there is a uniform bound on the Lipschitz constants of the generators of the group and this implies that the limit set of a finitely generated non-elementary Kleinian group is uniformly perfect.
\end{abstract}

\section{INTRODUCTION}

A rational semigroup $G$ is a semigroup of nonconstant rational functions defined on the Riemann sphere $\overline{\mathbb{C}}$ with the semigroup operation being functional composition. When a semigroup $G$ is generated by the functions $\left\{f_{i}: i \in I\right\}$, for some index set $I$, we write this as

$$
G=\left\langle f_{i}: i \in I\right\rangle
$$

Note that in [10] and [11] rational semigroups are always taken to have at least one element of degree at least two. We do not make such a restriction here.

The study of rational semigroups may be viewed as a generalization of the study of Kleinian groups, iteration of a rational function, and iterated function systems (IFS's). For example limit sets of Kleinian groups, Julia sets of a rational function, and self-similar sets generated by iterated function systems are Julia sets of rational semigroups (see Remarks 2.2 and 2.3) and in these sets fixed points are always dense (see [3], 4], and [12]).

It is known that the Julia set of a rational function is uniformly perfect. Several proofs of this fact have been given, namely by Eremenko [7], Hinkkanen [9], and Mañé and da Rocha [13. Also, Hinkkanen and Martin have shown in [11 that Julia sets of finitely generated rational semigroups are uniformly perfect when the semigroup is generated by maps all which have degree greater than or equal to two. (Although in 11] the theorem is stated under the weaker hypothesis that only one of the maps of the semigroup must have degree at least two, a closer inspection shows that the proof is only valid when all the maps are of degree at least two.) The proof uses the fact that Julia sets of single rational functions of degree at least two

Received by the editors August 26, 1998 and, in revised form, October 5, 1998.

1991 Mathematics Subject Classification. Primary 30D05, 58F23.

Key words and phrases. Rational semigroups, Kleinian groups, Julia sets, uniformly perfect, iterated function systems.

(C)2000 American Mathematical Society 
are uniformly perfect and also relies substantially on the fact that the semigroup is finitely generated. In this paper we show in Theorem 3.1 that when there exists a uniform bound on the Lipschitz constants with respect to the spherical metric associated with each generator of the rational semigroup the corresponding Julia set is necessarily uniformly perfect. This Lipschitz condition is trivially satisfied when the semigroup is finitely generated and so we see that this is an improvement on the previous result. Furthermore the proof does not rely on the fact that Julia sets of single rational functions are uniformly perfect and we also relax the restriction on the degree of the maps in the semigroup; thus we are able to apply this result to Möbius groups as well.

The author would like to thank the referee for the helpful comments.

\section{Definitions AND Basic FaCts}

In [10], p. 360, the definitions of the set of normality, often called the Fatou set, and the Julia set of a rational semigroup are as follows:

Definition 2.1. For a rational semigroup $G$ we define the set of normality of $G$, $N(G)$, by

$N(G)=\{z \in \overline{\mathbb{C}}:$ there is a neighborhood of $z$ on which $G$ is a normal family $\}$

and define the Julia set of $G, J(G)$, by

$$
J(G)=\overline{\mathbb{C}} \backslash N(G) .
$$

When $G=\langle f\rangle$, we abuse the notation and write $N(f)$ and $J(f)$ for $N(\langle f\rangle)$ and $J(\langle f\rangle)$ respectively.

Proposition 2.1 ([10], p. 360). The set $N(G)$ is forward invariant under each element of $G$ and $J(G)$ is backward invariant under each element of $G$.

The following proposition about the backward self-similarity of the Julia set has been noted by many people and is easy to prove.

Proposition 2.2. If $G=\left\langle g_{1}, \ldots, g_{N}\right\rangle$, then $J(G)=\bigcup_{i=1}^{N} g_{i}^{-1}(J(G))$ and $N(G)=$ $\bigcap_{i=1}^{N} g_{i}^{-1}(N(G))$.

The sets $N(G)$ and $J(G)$ are, however, not necessarily completely invariant under the elements of $G$. This is in contrast to the case of single function dynamics. For a discussion on completely invariant Julia sets the reader is referred to [19], [20] and [18].

Definition 2.2. We say that a rational semigroup $G$ is non-elementary if $J(G)$ has three or more points.

Lemma 2.1 (10, Lemma 3.1). The set $J(G)$ is perfect when $G$ is non-elementary.

Using an idea of Baker in [1, Hinkkanen and Martin have shown the following theorem. (The author has shown that the idea of Schwick in [17] can also be adapted to prove the following result.)

Theorem 2.A ([10], Theorem 3.1 and Corollary 3.1). If $G$ is a non-elementary rational semigroup, then the repelling fixed points of the elements of $G$ are dense in $J(G)$. Hence also

$$
J(G)=\overline{\bigcup_{g \in G} J(g)} .
$$


Remark 2.1. In [10] all the semigroups $G$ are assumed to have at least one element of degree two or more, but Lemma 2.1 and Theorem 2.A still hold (with the proofs given in the references) if we remove this restriction.

Remark 2.2. A Möbius group $G$, i.e., a group of maps of the form $(a z+b) /(c z+d)$ where $a d-b c \neq 0$, is a rational semigroup. If there does not exist a finite $G$-orbit in $\mathbb{R}^{3}$ (this is the definition of non-elementary in [3]), then one can see by [3] , p. 90 that $G$ is non-elementary in the sense of Definition 2.2. Whereas, if a Möbius group $G$ is non-elementary in the sense of Definition 2.2, then $G$ is non-elementary in the sense of [3] or $G$ is conjugate to a group of linear transformations (see [3], p. 84).

Since the limit set of a non-elementary Möbius group (which need not be discrete) is the closure of the set of repelling fixed points (see [3], p. 97) we see by Theorem 2.A that the notion of limit set and Julia set coincide in the case of non-elementary Möbius groups and, in particular, for non-elementary Kleinian groups, i.e., Möbius groups whose action is properly discontinuous at some point $z \in \overline{\mathbb{C}}$.

Remark 2.3. An iterated function system (IFS) generated by the (possibly infinite) set of contracting linear maps $\left\{g_{i}: i \in I\right\}$ on $\mathbb{C}$ (see [12]) corresponds to a rational semigroup generated by the inverses of the generating maps $\left\{g_{i}^{-1}: i \in I\right\}$. Note that the attracting fixed point of an element $g$ in the IFS is a repelling fixed point for $g^{-1}$ in the corresponding semigroup. Defining the attractor set $A$ for an IFS to be the closure of the attracting fixed points (see [12]), we see that $A$ is the Julia set for the corresponding rational semigroup. One may also note that when the IFS is generated by $\left\{g_{1}, \ldots, g_{n}\right\}$ the attractor set $A$ may be defined as the unique compact set $A \subset \mathbb{C}$ which satisfies $A=\bigcup_{i=1}^{N} g_{i}(A)$ and so it also follows from Proposition 2.2 that $A$ is the Julia set for the corresponding rational semigroup.

Example 2.1. Define the maps $g_{1}(z)=z / 3, g_{2}(z)=\left(e^{\pi i / 3} z+1\right) / 3, g_{3}(z)=$ $\left(e^{2 \pi i / 3} z+2\right) / 3$, and $g_{4}(z)=(z+2) / 3$. Then $J\left(\left\langle g_{1}^{-1}, g_{4}^{-1}\right\rangle\right)$ is the middle third Cantor set and $J\left(\left\langle g_{1}^{-1}, g_{2}^{-1}, g_{3}^{-1}, g_{4}^{-1}\right\rangle\right)$ is the von Koch curve (see [8], p. xv).

Definition 2.3. For a rational function $g$ of degree two or more and a point $z \in \overline{\mathbb{C}}$ we define the backward orbit with respect to $g$ by $O_{g}^{-}(z)=\{w$ : there exists $n$ such that $\left.g^{n}(w)=z\right\}$.

Definition 2.4. If $g$ is a rational function of degree two or more, we define the exceptional set of $g$ to be $E(g)=\left\{z \in \overline{\mathbb{C}}: O_{g}^{-}(z)\right.$ contains at most two points $\}$. If $g$ is a Möbius map with an attracting fixed point and a repelling fixed point, i.e., a loxodromic Möbius map, then we define the exceptional set $E(g)$ of $g$ to be the set consisting of the single attracting fixed point.

Proposition 2.3 (4], Theorem 6.9.4). Let $f$ be a rational function with $\operatorname{deg} f \geq 2$ or a loxodromic Möbius map. Let $W$ be a non-empty open set intersecting $J(f)$, and let $K$ be a compact subset of $\overline{\mathbb{C}} \backslash E(f)$. Then there exists an integer $N$ such that $K \subset f^{n}(W)$ for all $n \geq N$.

Definition 2.5. For a rational function $g$ we define $\operatorname{Lip} g=\inf \{M: \sigma(g(z), g(w)) \leq$ $M \sigma(z, w)$ for all $z, w \in \overline{\mathbb{C}}\}$ where $\sigma$ denotes the spherical metric.

Note that for a rational map $g$, we have Lip $g<+\infty$ as can be seen by proving the equivalent statement that $\frac{\left|g^{\prime}(z)\right|\left(1+|z|^{2}\right)}{1+|g(z)|^{2}}$ is bounded above on $\overline{\mathbb{C}}$ (see [4], p. 32). 
Definition 2.6. A conformal annulus is an open subset $\mathcal{A}$ of $\overline{\mathbb{C}}$ that can be conformally mapped onto the genuine annulus $\operatorname{Ann}\left(0 ; r_{1}, r_{2}\right)=\left\{z: 0 \leq r_{1}<|z|<r_{2} \leq\right.$ $\infty\}$ and the modulus of such a conformal annulus is given by

$$
\bmod (\mathcal{A})=\frac{1}{2 \pi} \log \frac{r_{2}}{r_{1}}
$$

We note that $\bmod (\mathcal{A})$ is a conformal invariant.

Definition 2.7. A conformal annulus $\mathcal{A}$ is said to separate a set $F$ if $F$ intersects both components of $\overline{\mathbb{C}} \backslash \mathcal{A}$ and $F \cap \mathcal{A}=\emptyset$.

Definition 2.8 (14, p. 192). We say that a compact subset $F \subset \overline{\mathbb{C}}$ is uniformly perfect if $F$ has at least two points and if there is a uniform upper bound on the moduli of all conformal annuli in $\overline{\mathbb{C}} \backslash F$ which separate $F$.

Uniformly perfect sets were introduced by A. F. Beardon and Ch. Pommerenke in 1978 in [2].

\section{THE MAIN RESUlT}

Theorem 3.1. Let $G=\left\langle g_{i}: i \in I\right\rangle$ be a non-elementary rational semigroup generated by the maps $\left\{g_{i}: i \in I\right\}$ such that $\sup _{i \in I}$ Lip $g_{i} \leq C<+\infty$. Then the Julia set $J(G)$ is uniformly perfect.

Proof. The proof given below is an adaptation of the proof that the Julia set of a single rational function of degree at least two is uniformly perfect which was presented in L. Carleson and T. Gamelin's book [6]. In this proof all notions of distance and convergence will be with respect to the spherical metric. Fix four points $z_{1}, z_{2}, z_{3}$ and $z_{4}$ in $J(G)$. Let $\delta>0$ be chosen small enough so that any two of the four selected points is at a (spherical) distance strictly greater than $C \delta$ from each other and such that $\delta<\frac{4 d}{5}$ where $d=\operatorname{diam}(J(G))$ denotes the spherical diameter of $J(G)$.

Suppose there is a sequence $\left\{A_{n}\right\}$ of conformal annuli in $N(G)$ with moduli tending to $\infty$ such that both components of $\overline{\mathbb{C}} \backslash A_{n}$ meet $J(G)$. Let $E_{n}$ be the component of $\overline{\mathbb{C}} \backslash A_{n}$ with the smaller spherical diameter. The diameter $\operatorname{diam}\left(E_{n}\right)$ tends to zero as can be seen by noting that that there are simple closed curves in $A_{n}$ whose hyperbolic lengths tend to 0 and which separate $E_{n}$ from $\overline{\mathbb{C}} \backslash\left(A_{n} \cup E_{n}\right)$. We assume that all $\operatorname{diam}\left(E_{n}\right)<\delta$.

Since $A_{n} \cup E_{n}$ is open and meets $J(G)$, we know by Theorem 2.A that there exists a point $z \in J(G) \cap\left(A_{n} \cup E_{n}\right)$ which is a repelling fixed point for the map $h \in G$, say. If $E(h) \cap J(G)=\emptyset$, where $E(h)$ denotes the set of (at most two) exceptional points of $h$, let $U=\emptyset$. Otherwise, let $U$ be a union of at most two open spherical discs centered at the points of $E(h) \cap J(G)$ each of diameter at most $\frac{d}{10}$ such that the boundary of each disk in $U$ contains a point of $J(G)$. Such disks can be found since $J(G)$ is perfect by Lemma 2.1. Let $K=J(G) \backslash U$ and note that $\operatorname{diam}(K) \geq \frac{4 d}{5}>\delta$. Then by the expanding property of Julia sets (Proposition [2.3) there exists a positive integer $k$ such that $h^{k}\left(A_{n} \cup E_{n}\right) \supset K$. Since $h^{k}\left(A_{n}\right) \subset N(G)$, which follows directly from Proposition [2.1] we conclude $h^{k}\left(E_{n}\right) \supset K$ and so $\operatorname{diam}\left(h^{k}\left(E_{n}\right)\right)>\delta$. We now let $f_{n} \in G$ be a function of minimal word length such that $\operatorname{diam}\left(f_{n}\left(E_{n}\right)\right)>\delta$. Each $f_{n}$ can be written in the form

$$
f_{n}=g_{i_{1}} \circ g_{i_{2}} \circ \cdots \circ g_{i_{m}}
$$


where each $i_{j} \in I$ depends on $n$. Letting $F_{n}=g_{i_{2}} \circ \cdots \circ g_{i_{m}}$ (we let $F_{n}=I d$ if $f_{n}$ is one of the generating functions in the set $\left.\left\{g_{i}: i \in I\right\}\right)$ we see by the minimality of the choice of $f_{n}$ that $\operatorname{diam}\left(F_{n}\left(E_{n}\right)\right) \leq \delta$. Hence $\operatorname{diam}\left(f_{n}\left(E_{n}\right)\right)=$ $\operatorname{diam}\left(g_{i_{1}}\left(F_{n}\left(E_{n}\right)\right)\right) \leq C \operatorname{diam}\left(F_{n}\left(E_{n}\right)\right) \leq C \delta$.

Let $\phi_{n}$ be a conformal map from the unit disk $\triangle$ onto $A_{n} \cup E_{n}$ such that $\phi_{n}(0) \in$ $E_{n}$. Note that here we use the fact that $J(G)$ is perfect to assert that $\overline{\mathbb{C}} \backslash\left(A_{n} \cup E_{n}\right)$ contains more than two points and therefore $A_{n} \cup E_{n}$ is conformally equivalent to the unit disk. Let $K_{n}=\phi_{n}^{-1}\left(E_{n}\right)$ and note that as the modulus of $\triangle \backslash K_{n}$ equals the modulus of $A_{n}$, we conclude as above that $\operatorname{diam}\left(K_{n}\right) \rightarrow 0$. Let $h_{n}=f_{n} \circ \phi_{n}$. Note that $h_{n}\left(\triangle \backslash K_{n}\right) \subset N(G)$ and $\operatorname{diam}\left(h_{n}\left(K_{n}\right)\right)=\operatorname{diam}\left(f_{n}\left(E_{n}\right)\right) \leq C \delta$. Hence each $h_{n}(\triangle)$ omits at least three of the points $z_{1}, z_{2}, z_{3}$ and $z_{4}$ in $J(G)$. Hence $\left\{h_{n}\right\}$ is normal in $\triangle$ by Montel's theorem. By the equicontinuity of the family $\left\{h_{n}\right\}$ we have $\operatorname{diam}\left(h_{n}\left(K_{n}\right)\right) \rightarrow 0$ since $\operatorname{diam}\left(K_{n}\right) \rightarrow 0$ and $0 \in K_{n}$ for all $n$. This is a contradiction since $\operatorname{diam}\left(h_{n}\left(K_{n}\right)\right)=\operatorname{diam}\left(f_{n}\left(E_{n}\right)\right)>\delta$.

Let $G^{\prime}$ be a Möbius group generated (as a group) by $\left\{g_{i}: i \in I\right\}$. Since the semigroup generated by $\left\{g_{i}: i \in I\right\} \cup\left\{g_{i}^{-1}: i \in I\right\}$ is $G^{\prime}$ and Lip $g_{i}=\operatorname{Lip} g_{i}^{-1}$ when $g_{i}$ is a Möbius transformation (see [4, p. 33) we see that Theorem 3.1 implies the following corollary.

Corollary 3.1. The limit set (Julia set) $J\left(G^{\prime}\right)$ of a non-elementary Móbius group is uniformly perfect when $\left\{g_{i}: i \in I\right\}$, the generators of $G^{\prime}$ (as a group), satisfy $\sup _{i \in I}$ Lip $_{i}<+\infty$.

We note that Kleinian groups which possess a uniform bound on the Lipschitz constants of its generators must necessarily be finitely generated since otherwise one can show that the group is not discrete (see [4], p. 33, or [3], p. 42). Hence Theorem 3.1 can only be used to duplicate the following result due to Pommerenke (see [15]). Pommerenke's proof is based on more analytic methods as opposed to the more geometric view used in this paper.

Corollary 3.2. The limit set (Julia set) $J\left(G^{\prime}\right)$ of a non-elementary Kleinian group is uniformly perfect when $G^{\prime}$ is finitely generated.

Remark 3.1. Not all limit sets of Kleinian groups are uniformly perfect. For each positive integer $n$ let $C_{n}=\left\{z:\left|z-a_{n}\right|=r_{n}\right\}$ and $C_{n}^{\prime}=\left\{z:\left|z-a_{n}-2 i\right|=r_{n}\right\}$ where the real numbers $a_{n} \searrow 0, r_{n} \searrow 0$ such that $r_{n}<a_{n} \leq 1$ and $\frac{a_{n}-r_{n}}{a_{n+1}+r_{n+1}} \rightarrow+\infty$. Then one can show that the Schottky group generated by the Möbius maps $g_{n}$ which map $C_{n}$ onto $C_{n}^{\prime}$ taking the interior of $C_{n}$ onto the exterior of $C_{n}^{\prime}$ has a limit set which is not uniformly perfect. One can see this by noting that the annuli $A_{n}=\left\{z: a_{n+1}+r_{n+1}<|z|<a_{n}-r_{n}\right\}$ separate the limit set.

Corollary 3.3. The attractor set $A$ of an IFS generated by the contracting linear maps $\left\{g_{i}: i \in I\right\}$ defined on $\mathbb{C}$ is uniformly perfect when $\sup _{i \in I}$ Lip $g_{i}<+\infty$.

\section{Applications}

Theorem 4.A (11], Theorem 4.1). Let $G$ be a rational semigroup such that $J(G)$ is uniformly perfect. Suppose that $z_{0}$ is a superattracting fixed point of an element $h \in G$. Let $A$ be the union of all the components of $N(h)$ in which the iterates of $h$ tend to $z_{0}$. Then either $z_{0} \in N(G)$ or $A \subset J(G)$. In particular, either $z_{0} \in N(G)$ or $z_{0}$ lies in the interior of $J(G)$. 
Corollary 4.1. Let $G=\left\langle g_{i}: i \in I\right\rangle$ be a non-elementary rational semigroup generated by the maps $\left\{g_{i}: i \in I\right\}$ such that $\sup _{i \in I}$ Lip $g_{i}<+\infty$ and let $z_{0}$ be a superattracting fixed point of some element of $G$. Then either $z_{0}$ lies in (the interior of) the Fatou set of $G$ or in the interior of the Julia set of $G$.

Julia sets of rational semigroups which contain elements of degree two or more are, however, not always uniformly perfect. In fact the following theorem is true.

Theorem 4.B ([11], Theorem 5.1). There exists an infinitely generated rational semigroup $G$ (all of whose elements have degree at least two) with the property that, for any positive integer $N$, the semigroup $G$ contains only finitely many elements of degree at most $N$, such that $J(G)$ is not uniformly perfect, and such that $G$ contains an element $g$ with a superattracting fixed point $\alpha$ with $\alpha \in \partial J(G) \subset J(G)$.

Example 4.1. Let $f_{0}$ be a non-constant rational function which has an attracting or superattracting fixed point at $\infty$. Pick a point $a \in J\left(f_{0}\right)$ and let $b_{n}$ be a sequence of points in $\mathbb{C}$ tending to $a$. Letting $f_{n}(z)=f_{0}\left(z+b_{n}\right)-b_{n}$, we can show that for the semigroup $G=\left\langle z^{2}, f_{n}: n \geq 0\right\rangle$ we have $\sup _{n \geq 0} \operatorname{Lip} f_{n}<+\infty$ and consequently $\{z:|z| \leq 1\} \subset J(G) \subsetneq \overline{\mathbb{C}}$.

Proof. Since the $b_{n}$ 's are bounded, one can easily show that there is a small neighborhood of $\infty$ which maps into itself by every element of $G$ and thus such a neighborhood must lie in $N(G)$. Also since the $b_{n}$ 's are bounded, one can show that $\sup _{n \geq 0}$ Lip $f_{n}<+\infty$ and so by Theorem $3.1 J(G)$ is uniformly perfect. Since $a-b_{n} \in J\left(f_{n}\right)$ for each $n$ we conclude that $0 \in \overline{\bigcup_{n=0}^{\infty} J\left(f_{n}\right)} \subset J(G)$ and so by Theorem 4 .A we have $\{z:|z| \leq 1\} \subset J(G)$.

One may ask whether there exists a finitely generated subsemigroup $G^{\prime}$ of $G$ in Example 4.1 such that $0 \in J\left(G^{\prime}\right)$. If so, one could use the weaker result of Hinkkanen and Martin in [11] (if the degree of $f_{0}$ is greater than or equal to two) to conclude that $J\left(G^{\prime}\right)$ is uniformly perfect and thus $\{z:|z| \leq 1\} \subset J\left(G^{\prime}\right) \subset J(G)$. It is often very difficult to accurately describe the Julia set of a rational semigroup and so the above is a difficult question. The author would like to know if there exists an example of a semigroup $G$ such that $J(G)$ contains a basin of attraction for some superattracting fixed point of some element of $G$ such that $J(G) \subsetneq \overline{\mathbb{C}}$, but also such that no finitely generated subsemigroup $G^{\prime}$ is such that its Julia set contains this basin of attraction.

There are many open questions regarding the description of Julia sets of rational semigroups that require further study in which theorems like Theorem 3.1 and Theorem 4.A may show to be useful. Some questions are the following: What conditions imply that $J(G)$ has nonempty interior? What conditions imply that $J(G)$ has empty interior? When does $J(G)$ have nonempty interior yet is a proper subset of $\overline{\mathbb{C}}$ ? In [22] and [21] Sumi uses an "open set condition" and the backward self-similarity (Proposition 2.2) to obtain some results in this direction and in particular shows that certain conditions imply $J(G)$ may be a generalized Cantor set, have no interior, have zero Lebesgue measure, or have Hausdorff dimension strictly less than 2. See also [16].

In order to get a feel for what certain Julia sets of finitely generated rational semigroups may look like one may use computer algorithms to draw these Julia sets by obtaining a measure whose support is exactly the Julia set. Of course, the 
usual warnings must be heeded as certain situations exist that seem to provide considerable barriers to the method giving an accurate picture (see [5]).

\section{REFERENCES}

[1] I. N. Baker. Repulsive fixed points of entire functions. Math. Z., 104:252-256, 1968. MR 37:1599

[2] A. F. Beardon and Ch. Pommerenke. The Poincarẽ metric of plane domains. J. London Math. Soc., 18:475-483, 1978. MR 80a:30020

[3] Alan F. Beardon. The geometry of discrete groups. Springer-Verlag, New York, 1983. MR 85d:22026

[4] Alan F. Beardon. Iterations of Rational Functions. Springer-Verlag, New York, 1991. MR 92j:30026

[5] David Boyd. An invariant measure for finitely generated rational semigroups. Complex Variables, to appear.

[6] Lennart Carleson and Theodore W. Gamelin. Complex Dynamics. Springer-Verlag, New York, 1993. MR 94h:30033

[7] A. Eremenko. Julia sets are uniformly perfect. Preprint, Purdue University, 1992.

[8] Kenneth Falconer. Fractal Geometry, Mathematical foundations and Applications. John Wiley and Sons, 1990. MR 92j:28008

[9] A. Hinkkanen. Julia sets of rational functions are uniformly perfect. Math. Proc. Camb. Phil., 113:543-559, 1993. MR 94b:58084

[10] A. Hinkkanen and G.J. Martin. The dynamics of semigroups of rational functions I. Proc. London Math. Soc.(3), 73:358-384, 1996. MR 97e:58198

[11] A. Hinkkanen and G.J. Martin. Julia sets of rational semigroups. Math. Z., 222(2):161-169, 1996. MR 98d:30038

[12] John E. Hutchinson. Fractals and self similarity. Indiana University Math. Journal, 30:731747, 1981. MR 82h:49026

[13] R. Mañé and L. F. da Rocha. Julia sets are uniformly perfect. Proc. Amer. Math. Soc., 116:251-257, 1992. MR 92k:58229

[14] Ch. Pommerenke. Uniformly perfect sets and the poincare metric. Arch. Math., 32:192-199, 1979. MR 80j:30073

[15] Ch. Pommerenke. On uniformly perfect sets and Fuchsian groups. Analysis, 4:299-321, 1984. MR 86e:30044

[16] Fu-Yao Ren. Advances and problems in random dynamical systems. Preprint, 1998.

[17] Wilhelm Schwick. Repelling periodic points in the Julia set. Bull. London Math. Soc., 29:314316, 1997. MR 97m:30029

[18] Rich Stankewitz. Completely invariant sets of normality for rational semigroups. Complex Variables. to appear.

[19] Rich Stankewitz. Completely invariant Julia sets of rational semigroups. PhD thesis, University of Illinois, 1998.

[20] Rich Stankewitz. Completely invariant Julia sets of polynomial semigroups. Proc. Amer. Math. Soc., 127:2889-2898, 1999. CMP 98:07

[21] Hiroki Sumi. On hausdorff dimension of Julia sets of hyperbolic rational semigroups. Kodai Math. J., 21:10-28, 1998. CMP 98:12

[22] Hiroki Sumi. On dynamics of hyperbolic rational semigroups. Journal of Mathematics of Kyoto University, to appear.

Department of Mathematics, Texas A\&M University, College Station, Texas 77843

E-mail address: richs@math.tamu.edu 